\title{
RIGID REALIZATIONS OF MODULAR FORMS IN CALABI-YAU THREEFOLDS
}

\author{
DOMINIK BUREK
}

\begin{abstract}
We construct examples of modular rigid Calabi-Yau threefolds, which give a realization of some new weight 4 cusp forms.
\end{abstract}

\section{INTRODUCTION}

The aim of this note is to construct new rigid Calabi-Yau realizations of weight 4 cusp forms as a resolution of a quotient of non-rigid examples listed by C. Mayer in 12 .

Recall that a smooth, projective threefold is called a Calabi-Yau threefold if it satisfies the following two conditions:

(i) The canonical bundle of $X$ is trivial and

(ii) $H^{1}\left(X, \mathcal{O}_{X}\right)=H^{2}\left(X, \mathcal{O}_{X}\right)=0$.

A Calabi-Yau threefold is said to be rigid if it has no infinitesimal deformations i.e. $H^{1}\left(X, \mathcal{T}_{X}\right)=0$. By Serre duality it is equivalent to the vanishing of $H^{2}\left(X, \Omega_{X}\right)$ or $h^{2,1}(X)=0$.

A lot of research on Calabi-Yau threefolds was motivated by the following conjecture:

Modularity conjecture. Any rigid Calabi-Yau threefold $X$ defined over $\mathbb{Q}$ is modular i.e. its $L$-series $L(X, s)$ coincides up to finitely many Euler factors with the Mellin transform $L(f, s)$ of a modular cusp form $f$ of weight 4 with respect to $\Gamma_{0}(N)$, where the level $N$ is only divisible by the primes of bad reduction.

In [5] and 6] L. Dieulefait and J. Manoharmayum proved the modularity conjecture for all rigid Calabi-Yau threefold defined over $\mathbb{Q}$ satisfying some mild assumptions on primes of bad reduction.

Finally the conjecture was obtained in [7 from the proof of Serre's conjecture given by C. Khare and J.-P. Wintenberger; see [10] and [11. However the question asked by B. Mazur and D. van Straten - which modular forms can be realized as a modular form of some rigid Calabi-Yau threefold, is still open.

Acknowledgments. This paper is a part of author's master thesis. I am deeply grateful to my advisor Sławomir Cynk for recommending me to learn this area and his help.

\section{Involutions of double octic Calabi-Yau threefolds}

Let $S \subset \mathbb{P}^{3}$ be an arrangement of eight planes such that no six intersect and no four contain a line. Then there exists a resolution of singularities $X$ of the double covering of $\mathbb{P}^{3}$ branched along $S$ which is a Calabi-Yau threefold.

A crepant resolution described in 4 is not uniquely determined but depends on the order of double lines. We can avoid this difficulty replacing the blow-up

2010 Mathematics Subject Classification. Primary 14J32; Secondary 11F03, 14J15.

Key words and phrases. Calabi-Yau threefolds. 
D. BUREK

of all double lines separately with the blow-up of their sum in the singular double cover. Now, by the universal property of a blow-up, every automorphism $\phi$ of the projective space $\mathbb{P}^{3}$ lifts to an automorphism $\widetilde{\phi}$ of the double octic $X$.

Any automorphism of a Calabi-Yau threefold acts on a canonical form $\omega$ of $X$ by a multiplication with a scalar $\omega \mapsto \mu \omega$. If the automorphism has finite order $n$ then $\mu^{n}=1$. We shall call an automorphism symplectic if it preserves the canonical form $\omega$ i.e. $\mu=1$.

We shall investigate in more details the case of an automorphism of order two. The fixed locus Fix $(f)$ of a symplectic involution of a Calabi-Yau threefold is a disjoint union of smooth curves, while the fixed locus of a non-symplectic involution is a disjoint union of smooth surfaces and isolated points.

2.1. Proposition. Let $\phi$ be an automorphism of the projective space $\mathbb{P}^{3}$ of order two such that

(i) the fixed locus of $\phi$ contains no double nor triple line of $S$,

(ii) planes intersecting in fourfold point are not invariant by $\phi$,

(iii) a fixed line of $\phi$ intersects $S$ with odd multiplicity in at most two points.

Then the fixed locus of the induced involution $\widetilde{\phi}: X \rightarrow X$ contains no curve with positive genus.

Proof. Our strategy is to perform the resolution and after each step verify that the fixed locus of the lifting of $\phi$ to the partial resolution contains no irrational curves. We start with the (singular) double covering of $\mathbb{P}^{3}$ branched along $S$. Any fixed curve of the involution must be mapped to a fixed line of $\phi$ in $\mathbb{P}^{3}$, if this curve is irrational then, by the Riemann-Hurwitz formula, the line intersects $S$ with odd multiplicity in at most four points. This contradicts the second assumption, which in fact is necessary.

The next step of the resolution is the blowing up of all fivefold points in the base of double covering, new branch divisor is the strict transform of $S$ plus the exceptional divisors. So any fixed curve of the involution is either birational to a fixed line in previous step or is a fixed line of the induced involution on a projective plane (isomorphic to an exceptional plane of the blow-up). At this step we introduce new double lines: intersections of the exceptional locus with strict transforms of the five planes.

The involution is not the identity on the $\mathbb{P}^{2}$ because otherwise we will get a fixed divisor in the double octic. If one of the double lines is fixed (dashed line $l_{1}$ in the picture below), then the fixed locus on $\mathbb{P}^{2}$ consists of $l_{1}$ and an isolated point. On the other hand on each line $l_{2}, \ldots, l_{4}$ we have at least two fixed points, hence the other four lines intersect, which is impossible.

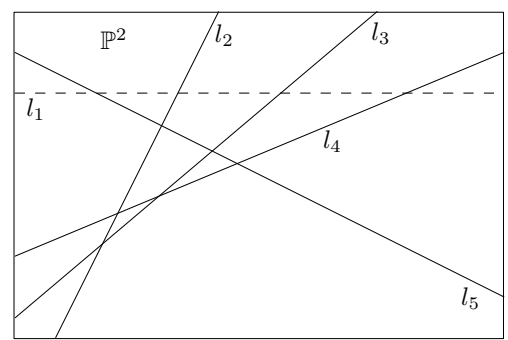

In the next step of resolution we blow up triple lines and as in the case of a fivefold point we add the exceptional divisors to the branch divisor. In the exceptional divisor we get the following configuration: 


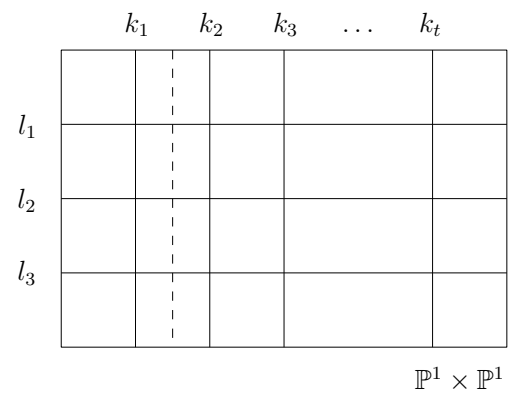

where $t$ denotes the number of fourfold points on that triple line.

Consequently any fixed curve is a vertical line (dashed line in the picture) and so it is rational. Again we introduce new double lines: $l_{1}, l_{2}, l_{3}$ and $k_{1}, k_{2}, \ldots$, $k_{t}$ (solid lines in the picture). Suppose that the line $k_{i}$ is fixed. Then we get four invariant planes: three planes containing $k_{i}$ and one transversal to them, which leads to the contradiction with assumption (ii)

Now, we blow-up all fourfold points, this time we do not add the exceptional divisors to the branch divisor. In the exceptional divisor over a fourfold point $P$ we get the following configuration:

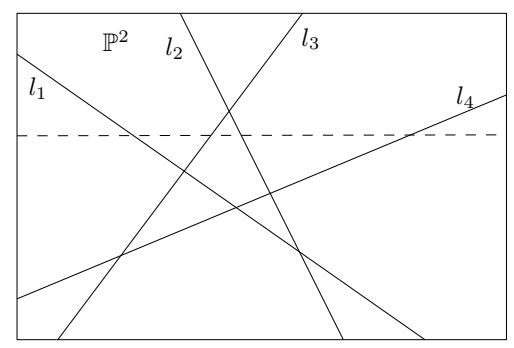

and in the double cover we get a double covering of $\mathbb{P}^{2}$ branched along the four solid lines. Any irrational fixed curve of the involution maps to a fixed line of involution on $\mathbb{P}^{2}$ (a dashed line in the picture), it is not of the other lines $l_{1}, \ldots, l_{4}$ because over each of them the double cover is an isomorphism.

In particular the involution on $\mathbb{P}^{2}$ is not identity, its fixed locus consists of the dashed line and an isolated point. As an involution of a projective line has two fixed points the lines $l_{1}, \ldots, l_{4}$ intersect and consequently the four planes passing through the fourfold point intersect along a line, which contradicts our assumptions.

Now the singular locus of the branch divisor is a union of double lines, some of them were introduced in the previous step, but none of them is fixed by the involution. In the last step we blow-up the sum of the double lines in the (singular) cover, a fiber of the blow-up is a line at a double point or a sum of three concurrent lines at a triple points. Since every fixed line is birational to a fixed line from the previous step of the resolution or a line (component of a fiber) the assertion of the proposition follows.

\section{MAIN RESULt}

In $12 \mathrm{C}$. Meyer gave a list of 63 one-parameter families of double octics $\left\{X_{\tau}\right\}_{\tau \in \mathbb{P}^{1}}$ with $h^{2,1}\left(X_{\tau}\right)=1$. Using an extensive computer search he found 18 examples in 11 families (see [12, table on page 54) such that for all primes $p \leq 97$ the trace of the Frobenius map Frob ${ }_{p}$ equals $a_{p}+p b_{p}$, where $a_{p}$ and $b_{p}$ are the coefficients of cusp forms $f_{4}$ and $f_{2}$ of weight 4 and 2 , respectively. This gives a strong numerical evidence of the modularity of $X$ in the following sense: the semi-simplification of the 
Galois representation on $H^{3}(X)$ decomposes into two-dimensional pieces isomorphic to Galois representations associated to $f_{4}$ and $f_{2}$. Equivalently, the $L$ series of $X$ factors as $L(X, s)=L\left(f_{4}, s\right) L\left(f_{2}, s-1\right.$ ) (see [8] and [9]). In fact modularity of all examples except Arr. no. 154 was proved in [3].

3.1. Remark. Different elements from Meyer's table with the same arrangement type are in fact isomorphic with a quadratic twist compatible with twists $\lambda$ in table [12. For an Arr. no. 4, from [2] we find that the map

$$
\left(\begin{array}{c}
x \\
y \\
z \\
t \\
u
\end{array}\right) \mapsto\left(\begin{array}{c}
A B y+A B z \\
-A B y \\
\left(-A^{2}+A B\right) x+\left(-A^{2}+A B\right) y-A^{2} z+\left(A^{2}-A B\right) t \\
\left(A B-B^{2}\right) t \\
A^{3} B^{3}(A-B)^{2} u
\end{array}\right)
$$

which gives an isomorphism (over $\mathbb{Q}$ ) between $X_{(A: B)}$ and the quadratic twist of $X_{(A-B: A)}$ by $-A(A-B)$. In particular $X_{(1:-1)}$ and $X_{(1: 2)}$ are isomorphic, they are also isomorphic to the quadratic twist of $X_{(2: 1)}$ by -2 . Since the cusp forms $32 \mathrm{k} 4 \mathrm{~A} 1$ and $32 \mathrm{~A} 1$ have $\mathrm{CM}$ by $\sqrt{-1}$ the quadratic twists by 2 and -2 coincide. Similar transformations exists also for Arr. no 13, 249 and 267.

3.2. Theorem. Let $X$ be a Calabi-Yau threefold from Meyer's table corresponding to Arr. no. 4, 13, 21, 53, 244, 267, 274. Then there exists a symplectic involution $\phi$ on $X$ and a crepant resolution $Y$ of a quotient $X / \phi$, which is a rigid, modular Calabi-Yau threefold.

Proof. By the remark 3.1 for each arrangement type in the theorem we can consider only one example. For all examples, we shall find a transformation $g: \mathbb{P}^{1} \rightarrow \mathbb{P}^{1}$, which has an isolated fixed point $\tau_{0}$ such that $g^{\prime}\left(\tau_{0}\right)=-1$ and isomorphisms $\widetilde{\phi_{\tau}}: X_{\tau} \rightarrow X_{g(\tau)}$ for which $\widetilde{\phi_{\tau_{0}}}$ is a symplectic involution satisfying conditions of the Proposition 2.1.

In the table Tab.1 we give an involution $\phi_{\tau}$ and the transformation $g$.

\begin{tabular}{c|c|c|l|c}
\hline Arr. no. & $g$ & $\tau_{0}$ & \multicolumn{1}{|c}{$\phi_{\tau}(x, y, z, t, u)$} & Cusp form \\
\hline \hline 4 & $1 / \tau$ & -1 & $\left(A y+A z,-A y, A x+A y, B t, A^{3} B u\right)$ & $32 \mathrm{k} 4 \mathrm{~A} 1$ \\
\hline 13 & $1 / \tau$ & 1 & $\left(B z, B y, B x, A t,-A B^{3} u\right)$ & $32 \mathrm{k} 4 \mathrm{~A} 1$ \\
\hline 21 & $(-1-\tau) / \tau$ & $-1 / 2$ & $\begin{array}{l}((A+B) x+(A+B) y,(-A-B) y, \\
\left.(A+B)^{3} B u\right)\end{array}$ & $32 \mathrm{k} 4 \mathrm{~B} 1$ \\
\hline 53 & $1 / \tau$ & 1 & $\left(A y, A x,-B t,-B z, A^{2} B^{2} u\right)$ & $32 \mathrm{k} 4 \mathrm{~B} 1$ \\
\hline 244 & $1 / \tau$ & -1 & $\left(B x, B y, A t, A z,-B^{2} A^{2} u\right)$ & $12 \mathrm{k} 4 \mathrm{~A} 1$ \\
\hline 267 & $1 / \tau$ & -1 & $(t,-z,-y, x, u)$ & $96 \mathrm{k} 4 \mathrm{~B} 1$ \\
\hline 274 & $1 / \tau$ & 1 & $(z,-t, x,-y, u)$ & $96 \mathrm{k} 4 \mathrm{E} 1$ \\
\hline
\end{tabular}

Tab. 1

Since $\tau_{0}$ is a fixed point of $g$ the weighted projective transformation $\phi_{\tau_{0}}$ induces an automorphism $\widetilde{\phi_{\tau_{0}}}$ of $X_{\tau_{0}}$ that transform $\omega$ to $\lambda \omega$ where $\lambda$ is the quotient of the determinant of the transformation on $\mathbb{P}^{3}$ by the coefficient in front of $u$. For each of the cases in the table Tab.1 we compute $\lambda=1$, so the involution $\widetilde{\phi_{\tau_{0}}}$ is symplectic. 
The action induced by $\widetilde{\phi_{\tau_{0}}}$ on $H^{1,2}\left(X_{\tau_{0}}\right)$ is given, via the Kodaira-Spencer map, by the multiplication by $g^{\prime}\left(\tau_{0}\right)$, thus $H^{1,2}\left(X_{\tau_{0}}\right)^{\left\langle\widetilde{\phi_{\tau_{0}}}\right\rangle}=0$. The quotient $X_{\tau_{0}} / \phi_{\tau_{0}}$ admits a crepant resolution $Y_{\tau_{0}}$ by blowing-up of double curves. From the special case of an orbifold formula (see [1]) we have the following formula

$$
H^{1,2}\left(Y_{\tau_{0}}\right) \simeq H^{1,2}\left(X_{\tau_{0}}\right)^{\left\langle\phi_{\tau_{0}}\right\rangle} \oplus \bigoplus_{C \in \operatorname{Fix}\left(\phi_{\tau_{0}}\right)} H^{0,1}(C),
$$

where $\operatorname{Fix}\left(\phi_{\tau_{0}}\right)$ consists of curves, which are fixed by the involution $\widetilde{\phi_{\tau_{0}}}$. Proposition 2.1 yields that $Y_{\tau_{0}}$ is a rigid Calabi-Yau manifold defined over $\mathbb{Q}$. From the involution we get a monomorphism $H^{3}\left(Y_{\tau_{0}}\right) \rightarrow H^{3}\left(X_{\tau_{0}}\right)$, so the modularity (and the cusp form) of $Y_{\tau_{0}}$ follows from the modularity of $X_{\tau_{0}}$.

3.3. Remark. According to our knowledge the last two examples in 3.2 give first rigid Calabi-Yau realizations of modular forms 96k4B1 and 96k4E1.

\section{REFERENCES}

[1] W. Chen, Y. Ruan, A new cohomology theory of orbifold, Comm. Math. Phys. 248(1), 1-31, 2004.

[2] S. Cynk, B. Kocel-Cynk, Classification of double octic Calabi-Yau threefolds, arXiv preprint arXiv:1612.04364

[3] S. Cynk, C. Meyer, Modularity of some non-rigid double octic Calabi-Yau threefolds, Rocky Mt. J. Math. 38, 1937-1958 (2008).

[4] S. Cynk, T. Szemberg, Double covers and Calabi-Yau varieties, Banach Center Publ. no. 44 (1998), pp. 93-101.

[5] L. Dieulefait, J. Manoharmayum, Modularity of rigid Calabi-Yau threefolds over $\mathbb{Q}$, CalabiYau varieties and mirror symmetry (Toronto, ON, 2001), 159166, Fields Inst. Commun. 38, Amer. Math. Soc. Providence, RI, 2003.

[6] L. Dieulefait, Improvements on Dieulefait-Manoharmayum and applications, preprint(2005), arXiv math/0508163

[7] F. Gouvêa, N. Yui, Rigid Calabi-Yau threefolds over $\mathbb{Q}$ are modular, Exp. Math. 29 (1) (2011), 142-149.

[8] K. Hulek, H. Verrill, On modularity of rigid and nonrigid Calabi-Yau varieties associated to the root lattice A4, Nagoya Math. Journal 179 (2005) 103-146.

[9] K. Hulek, H. Verrill, On the modularity of Calabi-Yau threefolds containing elliptic ruled surfaces, Mirror Symmetry V. AMS/IP Studies in Advanced Mathematics, vol. 38 (American Mathematical Society, Providence, 2006), pp. 19-34.

[10] C. Khare, J.-P. Wintenberger, Serre's modularity conjecture I, Invent. Math. 178 (2009), no. $3,485-504$

[11] C. Khare, J.-P. Wintenberger, Serre's modularity conjecture II, Invent. Math. 178 (2009), no. $3,505-586$

[12] C. Meyer, Modular Calabi-Yau threefolds, Fields Institute Monographs, 22. American Mathematical Society, Providence, RI, 2005.

Instytut Matematyki, Wydzią Matematyki i Informatyki, UniWersytet Jagielloński, ul. Łojasiewicza 6, 30-348 Kraków, Poland

E-mail address: dominik.burek@student.uj.edu.pl 